\title{
The representation of items in serial position
}

\author{
H. L. ROITBLAT, BENNETT POLOGE, and ROBERT A. SCOPATZ \\ Columbia University, New York, New York
}

\begin{abstract}
Four experiments with rats tested their ability to anticipate serial patterns made from elements of reward magnitudes $(14,7,3,1$, or 0 food pellets). Anticipation was measured by the running time in a straight alley. Elements arranged in a monotonically descending pattern were more easily anticipated than were the same elements arranged in a nonmonotonic pattern. Better anticipation was also observed when training utilized four trials per day with short interrun intervals (10-15 sec), spent in the startbox of the runway, than when training utilized one trial per day with long interrun intervals (4-5 min), spent in the rat's home cage. Anticipation of the monotonic sequence was also superior when training consisted of one trial per day with a short interrun interval relative to that observed with four trials per day and a long interrun interval. Following acquisition of anticipation of the monotonic sequence with a short interrun interval, transfer to the same sequence with a long interrun interval resulted in disruption of anticipation. Finally, anticipation of a well-learned monotonic sequence was not disrupted by replacement of individual rewarded elements in the sequence with a 0-pellet element. These experiments indicate that the duration between runs of a trial, but not that between trials or the number of trials per day, is important in the formation of serial expectancies. They also suggest that the rats come to represent the sequence as items in serial position.
\end{abstract}

A number of representational systems (Roitblat, 1982) have been proposed as the basis of rats' abilities to anticipate quantities of food reward in a serial anticipation task. In this type of task, rats are trained to run down a straight alley for a certain food reward whose magnitude varies systematically from run to run within a trial. Each food reward serves as one element in the sequence. The rat's running speed to an element is taken as an estimate of its expectation regarding the size of the anticipated element. Hulse and Campbell (1975), for example, compared the latencies (i.e., the time necessary to get from the start- to the goalbox) to each element $(0$, $1,3,7$, or 14 food pellets) in a sequence as a function of different serial arrangements of those elements. Running speed for those rats trained with a monotonic arrangement (0-1-3-7-14 or 14-7-3-1-0) varied with changes in the magnitude of the anticipated reinforcer. Other rats, trained with semirandom orderings of the same elements, which always terminated with 0 pellets for one group and 14 pellets for the other, failed to vary their running speed over elements in the series.

Hulse and Dorsky (1977) found that rats trained with a monotonic sequence, 14-7-3-1-0, learned to anticipate (i.e, run slowly to) the 0 -pellet element earlier than did rats trained with a weakly monotonic sequence, 14-5-5-1-0, or with a nonmonotonic se-

This research was supported by National Science Foundation Grant BNS 82-03017 and National Institute of Mental Health Grant 1-R01-MH37070. Bennett Pologe is now at Fordham University. Reprints may be obtained from H. L. Roitblat, Department of Psychology, Box 28 Schermerhorn Hall, Columbia University, New York, New York 10027. quence, 14-1-3-7-0. Identical results were obtained in a later experiment (Hulse \& Dorsky, 1979) with the monotonic sequences 18-10-6-3-0 or 18-10-6-3-1 versus the nonmonotonic sequences $18-3-6-10-0$ or 18 3-6-10-1. Again, the monotonic sequence was learned more quickly than the nonmonotonic sequence, as indexed by the decrease in running speed to the terminal element, whether it was 0 or 1 pellet.

The remarkable feature of these experiments is that different arrangements of the same elements make anticipation of those elements either easy or difficult. Furthermore, those sequences that facilitate anticipation are those that are formally simple in that they can be described by a simple monotonic rule of the form $E(i)>E(i+1)$. Recognizing this feature, Hulse and his associates have proposed a "pattern rule" representational system in which the rat is assumed to use features of the sequence's formal property (i.e., its monotonicity) to encode it as a pattern or rule. Anticipation, then, derives from application of the rule to the reward magnitude just experienced. As evidence for the importance of a generalized rule representation, Hulse and Dorsky trained rats with monotonic sequences that were two, three, or four elements in length, drawn from the set $0,1,3,5$, or 10 pellets. For one group, only monotonic sequences were presented. Although the particular element presented on a run varied from trial to trial, the pattern of the sequence remained constant: whatever the size of the element just obtained, the size of the next element would be smaller. Hence, strict paired associates between fixed elements were difficult to form, but knowledge of the monotonicity of the sequence would still allow anticipation on the basis of the pre- 
viously obtained element. Another group of rats were trained with the same elements used for the monotonic group, but these elements were presented in a random order. After training on these sequences for 70 trials, the subjects were transferred to either a new monotonic pattern of fixed length (16-9-3-1-0) or to a new nonmonotonic, but stable, sequence (16-1-39-0). The transfer task was learned most quickly by the group initially trained in the variable-length monotonic condition and transferred to the fixed-length monotonic condition. The slowest learning was obtained from the group initially trained on the monotonic sequence and then transferred to the nonmonotonic sequence. The two groups initially trained on the nonmonotonic sequence were intermediate in their acquisition of the transfer tasks.

In a conceptually related experiment (Fountain \& Hulse, 1981), rats were trained with either a strongly monotonic pattern of 14-7-3-1, a weakly monotonic pattern of 14-5-5-1, or a nonmonotonic pattern of 14-3-7-1 pellets. When a 0-pellet element was added to the end of the sequences, rats in the strongly monotonic sequence "extrapolated" the sequence by running even more slowly to the 0-pellet element on the first trial before ever having experienced it. While this result is interpretable in the context of rule-based anticipation, it may also be the result of the novel procedure and its associated novelty-induced inhibition. In other words, changing the procedure may simply have caused some rats not to run (or to run slowly) independent of any knowledge the rat might otherwise have regarding the sequence or its rules. Those rats that had learned the most about the sequence (i.e., the group trained with the strongly monotonic sequence), according to this hypothesis, were most disrupted by the change.

In contrast to the rule-based representational model proposed by Hulse and his associates, Capaldi and his associates have proposed a representational system in which the sequence is encoded as a set of stimulus-stimulus associations. The memory for each element in the series is assumed to serve as a cue signaling the next element. The signaling capacity of a stimulus depends on its association with a subsequent element and on generalized "strength" obtained as a function of its similarity to other elements in the sequence and the value of the element to which they are associated. Capaldi and his associates have argued that nonmonotonic sequences are more slowly learned because the second to the last element signaling 0 gains extra signaling strength from its similarity to other elements in the sequence which are followed by the subsequent presentation of nonzero elements.

In support of this associative account, Capaldi and Molina (1979) found that rats better anticipated the terminal 0-pellet element in either of the weakly mono- tonic sequences $15-15-0-0$ or 14-14-2-0 than in the monotonic sequence 15-10-5-0. They explained the difference between the strongly monotonic 15-10-5-0 sequence and the weakly monotonic 14-14-2-0 in terms of generalization and discriminability among the items in the sequences. The 5- and the 2-pellet elements are $\mathrm{S}-$ cues, which signal the nonrewarded runs of the sequence, whereas the 15-, 10-, and 14-pellet elements are $S+$ cues because they signal reward. Furthermore, they argued, the 15 - and the 10-pellet elements are more similar to the 5-pellet element than the 14-pellet elements are to the 2-pellet element. Therefore, the 5-pellet element receives more excitatory potential (i.e., ability to signal reward) from the 15- and the 10-pellet elements than the 2pellet element does from the 14-pellet elements. Discrimination is therefore better (and generalization lower) in the weakly monotonic sequence 14-14-2-0, and this is reflected in the greater increase in running time to the 0-pellet element relative to the others for the weakly monotonic than for the strongly monotonic sequence.

Similar arguments apply to the weakly monotonic sequence 15-15-0-0. In this sequence, the first 0-pellet element signals the terminal 0 -pellet element and is sufficiently different from the 15-pellet elements to avoid gaining generalized signal strength from them. Capaldi and Molina also note that the running time to the first 0-pellet element in this sequence is usually low, meaning that the rats did not anticipate it to be 0 . They argue that this failure of anticipation follows from the high signal strength of the 15-pellet element. This same element signals either 15 or 0 pellets. They do not present rules for combining expectancies of this sort, but practically any combination rule would result in high generalized signal strength being assigned to the 15-pellet elements.

Other experiments also demonstrate that element discriminability plays a role in serial anticipation learning (Capaldi, Verry, \& Davidson, 1980). Roitblat (1982), however, has shown that at least one version of the discrimination/generalization model proposed by Capaldi and his associates is incapable of accounting for the results observed by Hulse and his associates. Furthermore, as we understand the hypothesis, the discrimination/generalization model predicts that running speed in anticipation of the 1-pellet element should be differentiated more easily from the speed to the 14-pellet element in the nonmonotonic sequence 14-1-3-7-0 than in the monotonic sequence 14-7-3-1-0, because in the nonmonotonic sequence, 14 and the element most similar to 14 , that is, 7 , are both signalers of low reinforcer magnitudes on the subsequent run. They should both, therefore, have low signal strength and result in slower running to the 1- and 0-pellet elements. Although an analysis analogous to that presented by Roitblat (1982) con- 
firms this prediction, even when similarities among the other elements are taken into account, results of this sort have never been observed.

The apparent ability of the discrimination/generalization model to explain the results reported by Capaldi and his associates, its failure to account for the findings of Hulse and his associates, and the conflicting findings regarding the relative superiority of anticipation in monotonic, weakly monotonic, and nonmonotonic sequences suggests that two kinds of tasks are involved. Hulse (1980) has pointed to a number of differences between the procedures used in his laboratory and those used by Capaldi and his associates. Hulse asserted that Capaldi and his associates "have simplified their patterns-chiefly by making them shorter-such that rule structures either do not exist or are so impoverished that they no longer fairly and clearly represent serial patterns at all. Second, they have given their subjects relatively little practice and experience with the relevant stimuli when patterns do properly exist. Third, they have sometimes used very long, nonoptimal invervals between successive elements within a pattern, between successive pattern repetitions, or both" (Hulse, 1980, p. 689).

In this paper, we examine some of the differences between the procedures used to study serial anticipation learning that might account for the apparent differences obtained. In the process, we investigate some features of the memory representation used by rats in serial anticipation learning. We argue that neither rule encoding nor the discrimination/generalization hypothesis can account for the data.

\section{EXPERIMENT 1}

The most plausible source of the discrepancies between the findings of the two laboratories is differences in the details of the procedures used. For example, Hulse and Dorsky (1979) trained and tested their animals with 4 or 5 trials per day, for a total of at least 60 trials. They used a 10-15-sec interrun interval (IRI) and a 10-20-min intertrial interval (ITI). Capaldi and Molina (1979), in contrast, trained their animals with only a single presentation of the sequence per day, an IRI of 4-5 min, and a total of only 12 trials. In later experiments (Capaldi, Nawrocki, \& Verry, 1982; Capaldi \& Verry, 1981; Capaldi et al., 1980; Capaldi, Verry, \& Nawrocki, 1982), shorter IRI durations were used for more trials, but shorter sequences were used (see Hulse, 1980, p. 689).

Experiment 1 is intended to investigate the role played by the IRI duration and the number of trials per day on acquisition of serial anticipations.

\section{Method}

Subjects. The subjects were 20 male and female albino rats maintained at $85 \%$ ad-lib weight in individual cages under a light-dark cycle. All subjects in the experiments reported here had previously served as subjects in class exercises on operant conditioning for water reinforcers. They were all naive with regard to serial order training and food reward at the start of these experiments. Training for all subjects began at the same time.

Apparatus. Rats were tested in a straight alley $2 \mathrm{~m}$ long and $15 \mathrm{~cm}$ wide. A goalbox, $20 \mathrm{~cm}$ square, was located at one end of the runway, and a startbox of identical size was located at the other. Raising a guillotine door between the startbox and the runway triggered an electronic timer which was stopped when the rat entered the goalbox, breaking the beam of an electric eye. The goalbox was baited with 45-mg food pellets (Bioserv, Inc.).

Procedure. The rats were food-deprived over an 8-day period. On each of the first 5 days, they were handled for $10 \mathrm{~min}$. Pretraining began on the 6th day, during which they were handled for $5 \mathrm{~min}$ and then placed in the startbox and allowed to explore the runway for $5 \mathrm{~min}$. On the next 2 days of pretraining, the goalbox was baited with 10 pellets and the rats were allowed to explore the runway for $5 \mathrm{~min}$.

On the day following pretraining, the rats were assigned randomly to one of four groups of five rats each. All groups began serial anticipation training on the day following pretraining. Group 4-M-S received four presentations each day of the monotonic sequence 14-7-3-1-0 with a short interrun interval (IRI) of $10-15 \mathrm{sec}$ and an intertrial interval (ITI) of 20-25 min. Group 4N-S differed from Group 4-M-S only in that the nonmonotonic sequence 14-1-3-7-0 was used. Group 1-M-L received one presentation each day of the monotonic sequence with a long IRI of 4-5 min. Group 1-N-L was similarly trained, but with the nonmonotonic sequence. All groups received 60 trials.

For Groups 4-M-S and 4-N-S, the rat was placed in the startbox and the door was raised when the rat either put its nose to the door or scratched at it or, if neither of these behaviors occurred, after $30 \mathrm{sec}$. If the rat reached the goalbox within $60 \mathrm{sec}$, the latency was recorded, the rat was returned to the startbox after consuming the available food pellets, the arm was rebaited, if appropriate, and the next run was started. If the rat failed to reach the goalbox in the required time, it was returned to the startbox for the next run and a latency of $60 \mathrm{sec}$ was recorded. After the last run of the trial, the rat was returned to its cage and the next rat was given its trial. When all 10 rats in these two groups had received the first trial of the day, they were given the next trial in the same order. This procedure was repeated for the third and fourth trials of the day, thereby maintaining an ITI of 20-25 min for each rat.

The procedure for Groups 1-M-L and 1-N-L was similar to that used with Groups 4-M-S and 4-N-S, except that the rats were returned to their cages after each run. When all 10 rats in these two groups had received a given run of the trial, they were given the next run in the same order, resulting in an IRI of 4-5 min.

One rat was dropped from Group 4-N-S soon after the start of training because it became excessively vicious.

\section{Results}

Figure 1 displays the average running times to each element in the sequence averaged over the course of the entire experiment. Only Group 4-M-S showed anticipation of the 0-pellet element as indexed by slower running to it than to the other elements in the series. The extreme running times for Group 4-N-S are due to the fact that one rat in that group often took $30 \mathrm{sec}$ or more to reach the goalbox.

The data were analyzed in blocks of four trials. For Groups 4-M-S and 4-N-S, a block is 1 day's trials. For Groups 1-M-L and 1-N-L, a block is 4 days' trials. Each block of trials produced one running time for each rat for each element in the sequence. An analysis of variance (ANOVA) was conducted 


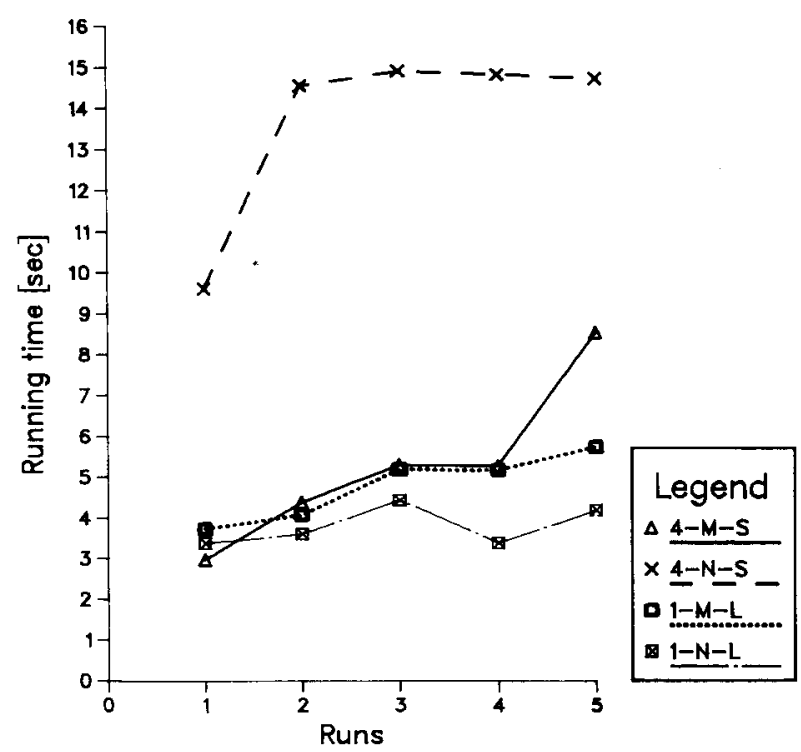

Figure 1. Average running times to each element in the four sequences used in Experiment 1. Group 4M-S was trained with four trials per day on a monotonic sequence with a short interrun interval. Group 4-N-S was trained with four trials per day on a nonmonotonic sequence with a short interrun interval. Group 1-M-L was trained with one trial per day on a monotonic sequence with a long intertrial interval. Group 1-N-L was trained with one trial per day on a nonmonotonic sequence with a long intertrial interval.

involving these running times as the dependent variable, procedure (4 trials/short IRI vs. 1 trial/long IRI) and pattern (monotonic vs. nonmonotonic) as between-groups factors, and runs (i.e., the successive elements in the sequence) and blocks as withingroups factors. This analysis revealed a significant effect of blocks $[\mathrm{F}(14,210)=5.33, \mathrm{p}<.001, \mathrm{MSe}=$ $191.862]$ and of runs $[F(4,60)=15.89, p<.001$, $\mathrm{MSe}=27.959]$. These main effects indicate simply that running times generally declined over the course of training and differed with the elements of the sequence $(4.663,6.227,7.060,6.749$, and $7.940 \mathrm{sec}$ for Elements 1 to 5, respectively). The main effects of procedure $[\mathrm{F}(1,15)=3.31, \mathrm{p}>.05, \mathrm{MSe}=2,892.644]$ and pattern $[\mathrm{F}(1,15)=1.69, \mathrm{p}>.05, \mathrm{MSe}=2,892.644]$, as well as their interaction $[\mathrm{F}(1,15)=2.70, \mathrm{p}>.05$, $\mathrm{MSe}=2,892.644$ ], failed to reach significance, indicating that average running times did not differ between the groups. Interactions were revealed between runs and procedure $[\mathrm{F}(4,60)=5.51, \mathrm{p}<.001, \mathrm{MSe}=$ 27.959], between runs and pattern $[F(4,60)=2.57$, $\mathrm{p}<.05, \mathrm{MSe}=27.959$ ], and between blocks and runs $[\mathrm{F}(56,840)=27.10751, \mathrm{p}<.001, \mathrm{MSe}=13.290]$.

The running times to the terminal 0-pellet element relative to the other elements in the sequence were tested by post hoc comparisons for each group. Group 4-M-S showed a significant difference $[F(4,840)$ $=18.4657, \mathrm{p}<.001$ ], running more slowly to the 0 -pellet element than to the other elements in the se- quence. Identical comparisons for the 4-N-S [F(4,840) $=1.4307, \mathrm{p}>.05], 1-\mathrm{M}-\mathrm{L} \quad[\mathrm{F}(4,840)=1.5749, \mathrm{p}>$ $.05]$, and $1-\mathrm{N}-\mathrm{L}[\mathrm{F}(4,840)<1]$ groups failed to reach significance.

\section{Discussion}

Consistent with earlier work involving the same sequences, the results of this experiment clearly indicate that the pattern in which elements are presented affects the ease with which rats anticipate the items. Furthermore, they show that two of the salient differences in procedure that have been used have important effects on rats' ability to anticipate patterns of elements. With five-element monotonic sequences, differential anticipation of the 0-pellet element is observed if the interrun interval is short and four repetitions of the pattern are presented per day (i.e., Group 4-M-S). The other combinations of pattern and procedure did not result in differential anticipation of the 0-pellet element. Hence, these results support assertions made by Hulse (1980) and by Roitblat (1982) that procedural differences between the laboratories of Hulse and of Capaldi make it difficult to compare results. When these differences are added to differences in the length of the pattern (Fountain, Evenson, $\&$ Hulse, 1983), it seems evident that different tasks are involved.

If, as is apparently the case, two serial anticipation tasks are being studied, then we might not expect the same theory (at least at the particular level at which the present theories are cast) to account for both. We want to emphasize that the apparent failure of the discrimination/generalization model to account for the data of the sort presented here and by Hulse and his associates does not render the model either useless or uninteresting. These results simply suggest a limitation on the domain to which the model applies, that is, it apparently does apply under conditions involving short sequences and relatively long interrun intervals. When patterns of the sort used by Hulse and his associates are trained, anticipation is easier when training occurs with short interrun intervals, four trials per day, and with monotonic sequences than with either long interrun intervals or nonmonotonic sequences.

\section{EXPERIMENT 2}

In Experiment 1, the length of the interrun interval (IRI) and the number of trials per day were confounded. The differences in the anticipation performance between Groups 4-M-S and 1-M-L could have been caused by either of these variables. In this experiment, we investigate the effects of one trial per day with a short IRI versus four trials per day with a long IRI on the ability of rats to anticipate items in a monotonic sequence. If the poorer anticipation observed in Group 1-M-L relative to that of Group 4- 
M-S is due to the duration of the IRI, then training with 1-M-S should result in better anticipation than training with 4-M-L. On the other hand, if the effect is due to trial spacing rather than to IRI duration, then better anticipation should be obtained with 4M-L than with 1-M-S.

\section{Method}

Apparatus. The apparatus was the same as that used in Experiment 1 .

Subjects. The subjects were eight male albino rats with pretraining histories similar to those of the subjects serving in Experiment 1.

Procedure. Pretraining was the same as that used in Experiment 1.

The four rats of Group 1-M-S received one trial of the monotonic sequence 14-7-3-1-0 per day with an IRI of 15-20 sec in a manner similar to that used with Group 4-M-S in Experiment 1. The four rats of Group 4-M-L received four presentations of the sequence each day with an IRI of 4-5 min and an ITI of 20-25 min in a manner similar to that used with Group 1-M-L in Experiment 1. Training continued for 60 trials.

\section{Results}

Figure 2 shows the running times to each element in the sequence averaged over the course of the experiment. Group 1-M-S showed differential anticipation of the 0-pellet element, but Group 4-M-L did not. An ANOVA, analogous to that conducted in Experiment 1 , was conducted involving the running times to each element in the sequence as the dependent variable, procedure as the between-groups factor (1-M-S vs. 4-M-L), and blocks and runs as withingroups factors. Again, each four trials produced one mean running time per element per rat. This analysis revealed a significant effect of blocks $[F(14,84)=$

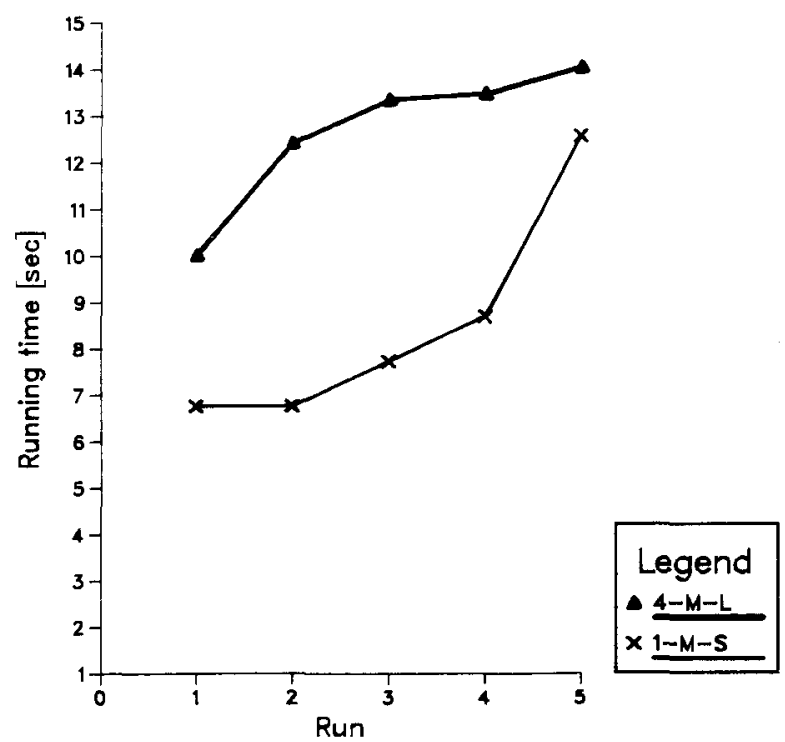

Figure 2. Running times to the elements of the two sequences used in Experiment 3. Group 4-M-L was trained with four trials per day on monotonic sequence with long interrun intervals. Group 1-M-S was trained with one trial per day on a monotonic sequence with short interrun intervals.
3.94, $\mathrm{p}<.001, \mathrm{MSe}=294.557]$ and of runs $[\mathrm{F}(4,24)$ $=11.60, \mathrm{p}<.001, \mathrm{MSe}=34.792]$ as well as interactions between runs and procedure $[F(4,24)=2.78$, $\mathrm{p}<.05, \mathrm{MSe}=34.792]$ and between blocks and runs $[\mathrm{F}(56,336)=1.41, \mathrm{p}<.05, \mathrm{MSe}=18.787]$. The lack of a main effect of procedure $[\mathrm{F}(1,6)<1, \mathrm{MSe}=$ $4,640.985]$ indicates that there was no reliable difference in average running time between the groups. Additionally, post hoc comparisons revealed that the running time to the 0 -pellet element was significantly slower than it was to the other elements for Group $1-\mathrm{M}-\mathrm{S}[\mathrm{F}(4,336)=16.532, \mathrm{p}<.001]$ but not for Group 4-M-L $[\mathrm{F}(4,336)=1.895, \mathrm{p}>.05]$.

\section{Discussion}

In combination with Experiment 1, this experiment showed that the duration of the interrun interval is crucial to the formation of adequate expectancies. When the interrun interval is short, but not when it is long, do the rats learn to anticipate a monotonic sequence by running slowly to the terminal 0 -pellet element. They suggest that the number of trials per day during training is not an important variable, but the time between runs of a trial is important. With sufficient time between runs, the rat apparently has time to forget the magnitude of the previously obtained element or to otherwise lose its place in the sequence.

\section{EXPERIMENT 3}

In Experiment 3, we explore the impact of long interrun intervals on the performance of an already learned sequence. If long interrun intervals interfere because they break up the pattern of the sequence, making it harder to detect, then they should have no effect on performance of an already acquired sequence. On the other hand, if long IRI durations interfere by making it more difficult for the rat to keep track of its current "location" in the sequence, then long IRI durations should interfere with performance as well as with acquisition.

Among the ways in which a long IRI could interfere with anticipation of a particular sequence is by interfering with the animal's ability to remember the immediately previous item. Both the rule-based and the discrimination/generalization models of serial anticipation learning suggest that to the extent that the sequence has been learned, each item is anticipated on the basis of the previously obtained item. For the rule-based model, acquisition of the sequence pattern does not depend on particular items' being consistently presented, but once acquisition has occurred, adequate performance requires that the previously obtained item be remembered in order to serve as the input to the "less than" rule; in order for $E(i+1)$ to be anticipated as less than $E(i)$, the rat must have information about $E(i)$. According to the discrimination/ 
generalization view, the memory for the previous item serves as a stimulus for the succeeding item. In either case, failure to remember the previous item should interfere with performance on the next item.

\section{Method}

The apparatus was the same as that used in Experiments 1 and 2. Immediately following the last day of Experiment 1, the rats from Group 4-M-S were tested for seven trials according to the procedure used for Group 1-M-L in Experiment 1. They were given one monotonic trial per day with a 4-5-min IRI spent in their home cages. In all other respects, the procedure was identical to that used in Experiment 1.

\section{Results and Discussion}

Running times to the 14-, 7-, 3-, 1-, and 0-pellet elements were $2.37,2.42,2.33,2.39$, and $2.80 \mathrm{sec}$, respectively. An ANOVA with trials and runs as within-groups factors revealed no significant differences among the runs $[\mathrm{F}(4,12)=1.08, \mathrm{p}>.05, \mathrm{MSe}=$ 1.2536]. The main effect of trials $[F(6,18)=1.22$, $\mathrm{p}>.05, \mathrm{MSe}=1.213 \mathrm{]}$ and the trials $\times$ runs interaction $[\mathrm{F}(24,72)<1, \mathrm{MSe}=0.749]$ also failed to reach significance. Either the long interrun interval or the return to the home cage during the interval interfered with the rat's ability to anticipate items in a known sequence. These data are consistent with an interpretation that suggests that acquisition of the sequence and performance on the sequence both depend on the rat's ability to keep track of its place in the sequence.

Honig (1978) has argued that memory functioning can fruitfully be divided into three categories. Learning in tasks such as those presented here, which involve a delay between the stimulus (e.g., the consumption of the previous element) and the response (e.g., running speed down the runway), is controlled by "associative memory," whose role is to maintain information about the stimulus until the consequence is obtained. Associative memory is needed, for example, to "bridge the gap" in trace conditioning between the end of the conditional stimulus and the beginning of the unconditional stimulus. Once a task is well learned, its stable properties are encoded in "reference memory." For example, in the present seria' anticipation task, the relationships between successive elements are constant from day to day and could be stored in reference memory. "Perception" of those interitem relations depends on the adequate functioning of associative memory, but once learned they are stored in reference memory. Finally, in addition to knowing about the relations between elements, the rat must keep track of its current position within the sequence. That is, even in a well-learned sequence, it is important to remember information about the previously obtained element(s) in order to determine which the next item will be. This information, which changes from run to run in the sequence, is said to be held in "working memory." Poor an- ticipation performance could follow either from a failure to remember which item follows which (reference memory) or from a failure to remember which item was most recently received (working memory). The poor performance of the rats on the well-learned sequence may have been due to a failure of reference memory, but was more likely due to interference produced by the long IRI on retention of the information in working memory. Similar interference with retention in Group 1-M-L in Experiment 1 prevented the formation of an adequate reference memory of the sequence.

Other explanations of the results of Experiment 3 are available. For example, one reason for the failure of anticipation may be due to the large procedural differences between the end of 4-M-S training and 1-M-L testing. These differences, such as being returned to the home cage during the interrun interval, may have so changed the task that the rats were unable to recognize it as the same one on which they had been trained. By this argument, the rats could remember perfectly well which item they had most recently received, but did not respond appropriately in the face of that knowledge. Although we cannot conclusively rule out such generalization decrement arguments, Experiment 4 suggests that that is not the case.

\section{EXPERIMENT 4}

Two hypotheses were discussed above, each of which attempts to explain the performance of rats on each run in a serial anticipation task as the reaction to the immediately preceding element. The ruleencoding hypothesis suggests that the animal applies a rule to the previous element to generate an ordinal prediction regarding the magnitude of the next element (i.e., whether it is smaller or larger). The discrimination/generalization hypothesis suggests that each element operates as a stimulus that signals the next item in the sequence. In either case, the basis for anticipation of a succeeding element is the element most recently received. The magnitude of other elements in the sequence may affect acquisition, discriminability, or the expected value of the elements, but the anticipation of each element is based solely on the element just obtained.

In Experiment 4, we examine rats' performance on the monotonic sequence when the food on a normally rewarded run is omitted. On probe trials, the normal 14-7-3-1-0 sequence is replaced by $0-7-3-1-0$, $14-0-3-1-0,14-7-0-1-0$, or 14-7-3-0-0. According to the discrimination/generalization view, this manipulation should adversely affect performance, since it is a break in the associative chain (Capaldi et al., 1980). Not only does the wrong reward appear, but the animal is left with an inappropriate stimulus to dictate its performance on the next run because 0 pel- 
lets has always signaled the end of the trial. A rulebased model also implies that altering a run of the sequence will have some effect on performance, because the modified sequence has no consistent rule structure. Both models, then, predict that replacing an element in a monotonic sequence with a novel 0pellet element should be disruptive of subsequent running speed.

\section{Method}

Apparatus. The apparatus was the same as that used in Experiments 1,2 , and 3 .

Subjects. The subjects were five rats from Experiment 1. Two were in the original 4-M-S group and also served in Experiment 3, one was in the 1-M-L group, and two were in the 1-N-L group.

Procedure. All rats were trained on the 4-M-S schedule. The two rats from Group 4-M-S in Experiment 1 were retrained for 2 days ( 8 trials) following their 7 trials on the 1-M-L schedule in Experiment 3. The other three rats were retrained for 60 trials. At the end of retraining, all animals were running quickly to the nonzero elements and slowly to the terminal 0-pellet element.

Testing began on the day immediately following the last day of retraining. On the third trial of the day, the food on one reinforced run of the sequence was omitted. Thus, on each day, each rat received two control trials of 14-7-3-1-0 followed by a probe trial of 0-7-3-1-0 (M14), 14-0-3-1-0 (M7), 14-7-0-1-0 (M3), or 14-7-3-0-0 (M1), and then a final control trial. The order of the probe trials was randomized for each rat. When a rat had received the four different probe trials, a new random order was created for that rat. Testing continued for $\mathbf{1 6}$ days or four times through the four probe trials.

\section{Results}

The results of this experiment are shown in Figure 3. M14 refers to trials on which the 14-pellet element was replaced by a 0-pellet element, M7 refers to trials on which the 7-pellet element was replaced, and so forth. The important data in the figure are the running times to the element following the replaced element. It is apparent that replacing an element had no effect. An ANOVA on the running times to each element in the sequence (for each trial) with trial type and runs as within-groups factors revealed that running times to the elements in the sequence differed $[F(4,16)=15.03, p<.001, M S e=22.022]$, but trial type (i.e., control trials and each sequence with a replaced element: $M 14, M 7, M 3$, and $M 1$ ) did not differ $[F(4,16)=1.23, p=.3389, \mathrm{MSe}=5.965]$, and nor was an interaction found between runs and trial type $[F(16,64)=1.42, p=.1612, M S e=6.061]$.

\section{Discussion}

These data clearly show that omission of a reinforcer had no effect on performance. If the run following the probe run was reinforced, they ran fast; if it was not, they ran slowly. The apparent differences seen in Figure 3 among the running times to the terminal 0-pellet element did not reach significance (i.e., the trial type $\times$ runs interaction was not significant). In any event, these differences are not ordered with respect to the element that was replaced for that

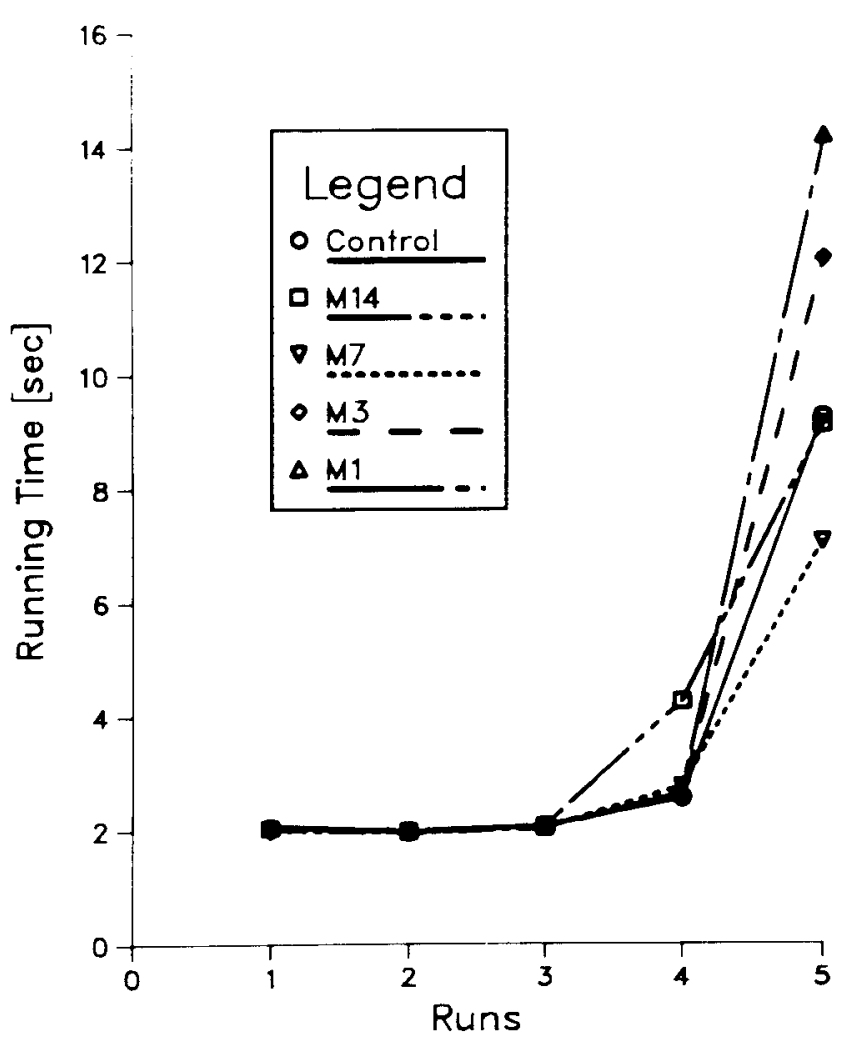

Figure 3. The running times to each element in the sequences tested in Experiment 4. On control trials, the sequence 14-7-3-1-0 was presented; on M14 trials, the 14-pellet element was replaced by 0 pellets; and on M7 trials, the 7-pellet element was replaced, etc.

trial type. Hence, they most likely represent chance variability in the running speeds between trials.

These data are inconsistent with representational systems based on either rule-learning or simple itemto-item associative mechanisms. Both of these models argue that the controlling properties of a given run of the sequence dictate performance on the subsequent run. Our data indicate instead, that the rats are representing the sequence as items in their serial position. Such a representation is not dependent on associative mechanisms or uninterrupted monotonicity. Performance on the sequence, once learned, is therefore quite robust and remains impervious to any distraction from missing elements.

\section{GENERAL DISCUSSION}

As in previous studies reported by Hulse and his associates, the order in which the stimuli are presented affects the ease with which those elements are anticipated. Monotonic orderings are more easily anticipated than are nonmonotonic orderings of the same elements. We also found that the time between runs of a trial, but not the time between trials (or the number of trials per day), affects the ease with which a 
series is anticipated. This effect of the interrun interval duration occurred even in Experiment 3, in which the animals had already been well trained and were previously anticipating the sequence.

Roitblat (1982) argued that sufficient procedural differences existed which made comparison of data obtained by Capaldi and his associates with those obtained by Hulse and his associates problematical. The experiments presented here confirm that assertion. When temporal parameters similar to those used by Capaldi et al. (1980), for example, are employed, they interfere with rats' ability to anticipate items in a monotonic sequence. IRI durations that were adequate for anticipation of shorter sequences, such as 14-14-2-0 and 1-29-0 (Capaldi \& Molina, 1979), were too long for anticipation of longer sequences such as those tested in the present study. Furthermore, they were too long for both acquisition and performance on the sequences as seen in Experiments 2 and 3 . The sequences studies by Capaldi and Molina also differed from the sequences found in the present study to be most easily anticipated in the complexity of their patterns and in the magnitude of the differences among the elements. The performance they observed, in combination with the results presented here, suggests that there might be an interaction among pattern length, pattern complexity, and IRI duration. Such an interaction between pattern length and pattern complexity has been reported by Fountain et al. (1983). They found that rats learned long and short monotonic sequences in fewer trials than long and short nonmonotonic sequences (18$1-0$ and 18-10-6-3-1-0 vs. $1-18-0$ and $10-1-3-6-18-0$ ). They also observed that short nonmonotonic sequences were learned faster than long nonmonotonic sequences, but no differences were observed in the speed of acquisition of short versus long monotonic sequences. This finding suggests that short and long sequences are learned differently from one another. We can speculate that tests of performance following acquisition of those sequences, such as those presented here, would also reveal differences.

Furthermore, Roitblat (1982) showed that a rigorous interpretation of the model proposed by Capaldi and his associates requires that, in order for less differentiation between the 14- and the 0-pellet elements to be observed in the nonmonotonic than in the monotonic conditions, more differentiation between the 14- and the 1-pellet elements must be observed in the nonmonotonic than in the monotonic condition. In contrast to this prediction, less differentiation of running times to all elements is observed in nonmonotonic than in monotonic sequences. We can only conclude that the generalization/discrimination model proposed by Capaldi and his associates is inadequate to deal with the sort of data presented here. As Hulse and Roitblat asserted, the procedural differences between the two tasks coming from the two laboratories makes comparison of their data problematical.

By the same token, neither the model advocated by Hulse and his associates nor the model described in the present study can do an adequate job in accounting for the data typically obtained by Capaldi and his associates. Although both sets of tasks have many common features, Experiments 1 and 2 show that they do differ in important ways. Apparently, there are at least two types of task being studied. It may be premature to expect that a single theory can account successfully for both types.

Experiments 1-3 of the present study demonstrate the importance of the duration of the interrun interval for both the acquisition and performance of serial anticipation tasks. It appears that the IRI duration affects not only what the animal learns from a situation, but also how the animal performs. Aside from simply forgetting information about the previous element(s) in the series (or about the current location within the series), at least three other explanations are available for the effects of interrun interval duration on performance on an already learned series. As was suggested above, performance could be poorer with long than with short interrun intervals because the change in interrun interval makes the series unrecognizable. Alternatively, the rats could have learned to anticipate the series on the basis of time since the start of the trial. Extending the IRI duration destroyed the relationship between time and items, and so made anticipation more difficult. The 0 -pellet element simply did not occur at the anticipated time. Finally, placing the rats in their home cages during the IRI may have caused them to reset their memories to the beginning of the series because removal of the rat from the cage had always reliably predicted the 14pellet element in the past.

Of these three potential explanations, only the last seems at all reasonable. If, according to the generalization decrement explanation, the increase in the duration of the interrun interval was sufficient to render the series in Experiment 3 unrecognizable, one should certainly expect that explicit manipulation of the primary stimuli would have a similar effect. Instead, replacing a nonzero element with 0 pellets during Experiment 4 had no effect. It simply does not seem plausible that manipulation of an incidental variable could have a larger effect when manipulation of an explicitly central variable, one that had been shown in other experiments to be potent in affecting anticipation, did not.

Anticipation on the basis of time since the start of the trial is consistent with the results obtained in Experiment 4, but is unlikely in light of the differences observed between the monotonic and nonmonotonic groups in Experiment 1. In both types of sequences, the 0-pellet element could occur at the same relative time. The same number of runs and the 
same number of pellets precede the terminal 0-pellet element in the two groups, yet it is the arrangement of those elements that determines whether that terminal element will be anticipated or not. Hulse (1978) presents similar arguments.

Finally, it is possible that it is not the long duration between runs that interferes with performance, but rather the fact that long IRIs were spent in the home cage whereas short IRIs were not. In fact, these experiments are not sufficient to differentiate between the resetting versus the decay of working memory. While this distinction is itself interesting, the conclusion that long IRIs spent in the home cage interfere with memory regarding the sequence is sound.

These experiments provide us with information regarding the representation used by rats in serial anticipation learning. Among the alternatives are (1) paired associates, (2) patterns, rules, or transformations, and (3) lists.

Hulse (1978) suggested that " a hypothesis based on the premise of associative chaining easily accounts for the Hulse and Campbell data"' (p. 322). Capaldi and his associates applied Capaldi's extensive work on effects of sequential reinforcer patterns to explain these results (e.g., Capaldi et al., 1980). As they recognized, a simple associative mechanism would be too powerful in that it would predict that nonmonotonic sequences would be anticipated as easily as monotonic sequences made up of the same elements. The discrimination/generalization model limits power by including a mechanism for transmitting generalized associative "strength" to similar valued items (see also Self \& Gaffan, 1983).

Of primary importance to the paired-associate account is the position that rats "employ the memory of one reward event as a cue to signal the next reward event of the series"' (Capaldi et al., 1980, p. 575). If this were the basis for their responding, then omission of reward, as described in Experiment 4, should have adversely affected performance. By leaving the goalbox unbaited on what was supposed to be a rewarded run of the sequence, we altered the memory of the previous run, and we left the rat with an inappropriate cue for the following run, since 0 pellets was always the last run of the sequence. The rats, however, were wholly unaffected by this disruption. They continued to run fast on rewarded runs and slowly on the terminal nonrewarded run.

Rule-encoding is the representational system most favored by Hulse and his associates. It also fails, however, to explain the results obtained in Experiment 4. If a rat's knowledge of the sequence is in the form of the rule $E(i)>E(i+1)$, then breaking that rule should have some effect on performance. No effect was observed. Performance on the probe trials was indistinguishable from performance on the control trials.
Finally, Hulse (1978) draws a parallel between the performance of rats on serial anticipation tasks and the performance of humans on serial pattern-learning tasks. While these tasks share some similarities, one must be cautious in interpreting the parallel, because at least two different methods are used to study humans' memory for serial patterns. In one type of task, humans are typically given a sequence consisting of a small set of ordered elements which conform to a particular rule or pattern. They are then asked to extend the pattern by selecting another, or a subsequent, group that also conforms to the sample pattern (e.g., Simon \& Kotovsky, 1963). The experiments described here, however, appear to be more similar to the other type of task in which subjects are asked to memorize and reproduce a patterned list. In these list-learning experiments with human subjects, organized or structured lists are typically found to be more easily remembered than unorganized lists (Mandler, 1967). Jones (1974) presents a more complete review of serial pattern learning by humans of both types of task.

List learning by humans does not appear to result in the formation of paired associates between subsequent list items (Restle \& Brown, 1970; Young, 1962). In fact, pretraining for learning a list by presenting the items in pairs has been found to interfere with later list learning (Young, 1959). Similarly, Straub and Terrace (1982) found that pretraining pigeons with paired subsets of a simultaneous chain did not facilitate acquisition of the entire chain. Apparently, in these list-learning experiments, humans and pigeons form more sophisticated representations of the list in which each item is represented in combination with more items than the preceding and succeeding elements as items in serial position. Experiment 4 suggests that rats in the present study similarly represented the sequences of reinforcer magnitudes as items in serial position. As Capaldi (1982, p. 373) noted in commenting on Roitblat's (1982) review of serial anticipation learning, "What is required in addition to (or besides) the rule is some other representation(s) of which there are many, such as associating each event with positional cues (e.g., Young, 1962)."

We wish to emphasize that the concept of items in serial position applies to a fully learned sequence. It is not a model of the learning process but of how the sequence is represented in memory after it is learned. One way in which structural complexity may play a role in acquisition is via the correlation inherent in a structurally simple sequence between item magnitude and serial position. Given the apparent finding that rats represent sequences of the sort used here as items in serial position, it becomes clear that serial position is a more important feature of lists than simply the necessary result of presenting only 
one item at a time. Instead, it is a salient feature of the task. We can speculate that formally simpler sequences are more easily learned because, to the extent that the rat learns about either the serial position or the items in that position, that knowledge transfers partially from one to the other. Nonmonotonic sequences do not contain this redundancy, and so both items and serial positions have to be learned separately.

Similarly, we do not doubt that associative mechanisms play a role in the early stages of pattern learning. Given the proper conditions, however, such as a short retention interval and structural simplicity, it is clear that other, higher order learning takes place.

\section{REFERENCES}

Capaldo, E. J. Memory and rules in animal serial learning. $B e$ havioral and Brain Sciences, 1982, 3, 373.

Capaldi, E. J., \& Molina, P. Element discriminability as a determinant of serial pattern learning. Animal Learning \& Behavior, 1979, 7, 318-322.

Capaldi, E. J., Nawrocki, T. M., \& Verhy, D. R. Difficult serial anticipation pattern learning in rats: Rule-encoding vs. memory. Animal Learning \& Behavior, 1982, 10, 167-170.

Capaldi, E. J., \& Verry, D. R. Serial order anticipation learning in rats: Memory for multiple hedonic events and their order. Animal Learning \& Behavior, 1981, 9, 441-453.

Capaldi, E. J., Verry, D. R., \& Davidson, T. L. Memory, serial anticipation learning, and transfer in rats. Animal Learning \& Behavior, 1980, 8, 575-585.

Capaldi, E. J., Verry, D. R., \& Nawrocki, T. M. Multiple hedonic memory: Memory for more than one hedonic event in rats. Animal Learning \& Behavior, 1982, 10, 351-357.

Fountain, S. B., Evensen, J. C., \& Hulse, S. H. Formal structure and pattern length in serial pattern learning by rats. Animal Learning \& Behavior, 1983, 11, 186-192.

Fountain, S. B., \& Hulse, S. H. Extrapolation of serial stimulus patterns by rats. Animal Learning \& Behavior, 1981, 9, 381-384.

HonIG, W. K. Studies of working memory in the pigeon. In S. H.
Hulse, H. Fowler, \& W. K. Honig (Eds.), Cognitive processes in animal behavior. Hillsdale, N. J: Erlbaum, 1978.

Hutse, S. H. Cognitive structure and serial pattern learning by animals. In S. H. Hulse, H. Fowler, \& W. K. Honig (Eds.), Cognitive processes in animal behavior. Hillsdale, N.J: Erlbaum, 1978.

Hulse, S. H. The case of the missing rule: Memory for reward vs. formal structure in serial pattern learning. Animal Learning \& Behavior, 1980, 8, 689-690.

Hulse, S. H., \& Camprell, C. E. “Thinking ahead" in rat discrimination learning. Animal Learning \& Behavior, 1975, 3, 305-311.

Hulse, S. H., \& Donsky, N. P. Structural complexity as a determinant of serial pattern learning. Learning and Motivation, $1977,8,488-506$.

Hulse, S. H., \& Donsxy, N. P. Serial pattern learning by rats: Transfer of a formally defined stimulus relationship and the significance of nonreinforcement. Animal Learning \& Behavior, 1979, 7, 211-220.

Jones, M. R. Cognitive representations of serial patterns. In B. Kantowitz (Ed.), Human information processing: Tutorials in performance and cognition. Hillsdale, N.J: Erlbaum, 1974.

Mandle R, G. Organization and memory. In K. W. Spence \& J. T. Spence (Eds.), The psychology of learning and motivation (Vol. 1). New York: Academic Press, 1967.

Restle, F., \& Brown, E. R. Serial pattern learning. Journal of Experimental Psychology, 1970, 83, 120-125.

Roitblat, H. L. The meaning of representation in animal memory. Behavioral and Brain Sciences, 1982, 5, 353-406.

Self, R., \& Gaffan, E. A. An analysis of serial pattern learning by rats. Animal Learning \& Behavior, 1983, 10, 10-18.

Simon, H. A., \& Kotov8Ky, K. Human acquisition of concepts for sequential patterns. Psychological Review, 1963, 70, 534-546.

Straub, R. O., \& Terrace, H. S. Generalization of serial learning in the pigeon. Animal Learning \& Behavior, 1982, 9, 454-468.

Young, R. K. A comparison of two methods of learning serial associations. American Journal of Psychology, 1959, 74, 554-559.

Young, R. K. Tests of three hypotheses about the effective stimulus in serial learning. Journal of Experimental Psychology, $1962,63,307-313$.

(Manuscript received March 21, 1983; revision accepted for publication August 8, 1983.) 